\title{
Aplicaciones prácticas de la espectroscopía de absorción infrarroja en el estudio de los crudos, del clínker y del cemento portland anhidro
}

ToMAs VAZQLEZ, Dr. en Ciencias Quimicas

En la primera parte de este trabajo (*) se trató brevemente y de una manera fundamentalmente práctica el estudio por espectroscopía infrarroja de calizas, arcillas, clínker portland y sus fases constituyentes. En el presente trabajo finalizará esta exposición con una visión general de algunas posibilidades que tiene la espectroscopía IR en el estudio del cemento portland anhidro, así como el estudio breve de puzolanas, escorias y cenizas volantes, como adiciones activas.

\section{ESTUdio Del CEMENTO PORTLANi POR IR}

Como ya se ha dicho, el espectro IR de una mezcla de sustancias es equivalente a la super-. posición de los espectros aislados.

Así el espectro IR de un cemento portland supone la superposición de los espectros aislados del clínker y del yeso adicionado.

\subsection{Yeso dihidrato, hemihidrato, anhidrita y singenita}

La i.dentificación del regulador de fraguado (supuestamente $\mathrm{CaSO}_{4} .2 \mathrm{H}_{2} \mathrm{O}$ ) integrante del cemento es de interés para prever comportamientos anómalos (principalmente en el fraguado), a consecuencia bien de una parcial deshidratación del yeso para dar hemihidrato, $\mathrm{CaSO}_{4} \cdot 1 / 2 \mathrm{H}_{2} \mathrm{O}$, bien de la presencia de anhidrita $\mathrm{CaSO}_{4}$, o incluso de singenita, $\mathrm{CaSO}_{4}$. . $\mathrm{K}_{2} \mathrm{SO}_{1} \cdot \mathrm{H}_{2} \mathrm{O}$.

Dado que tanto el yeso dihidrato, como el hemihidrato, la anhidrita y la singenita tienen espectros IR suficientemente diferenciables (fig. 1-a, cuadro 1), la identificación correspondiente es inmediata. En la fig. 1-b se dan los espectros de un clínker portland que contiene yeso, hemihidrato y singenita, respectivamente.

(*) Publicada en MATERIAles DE CONSTRUCCiON núms. 175 y 177. 

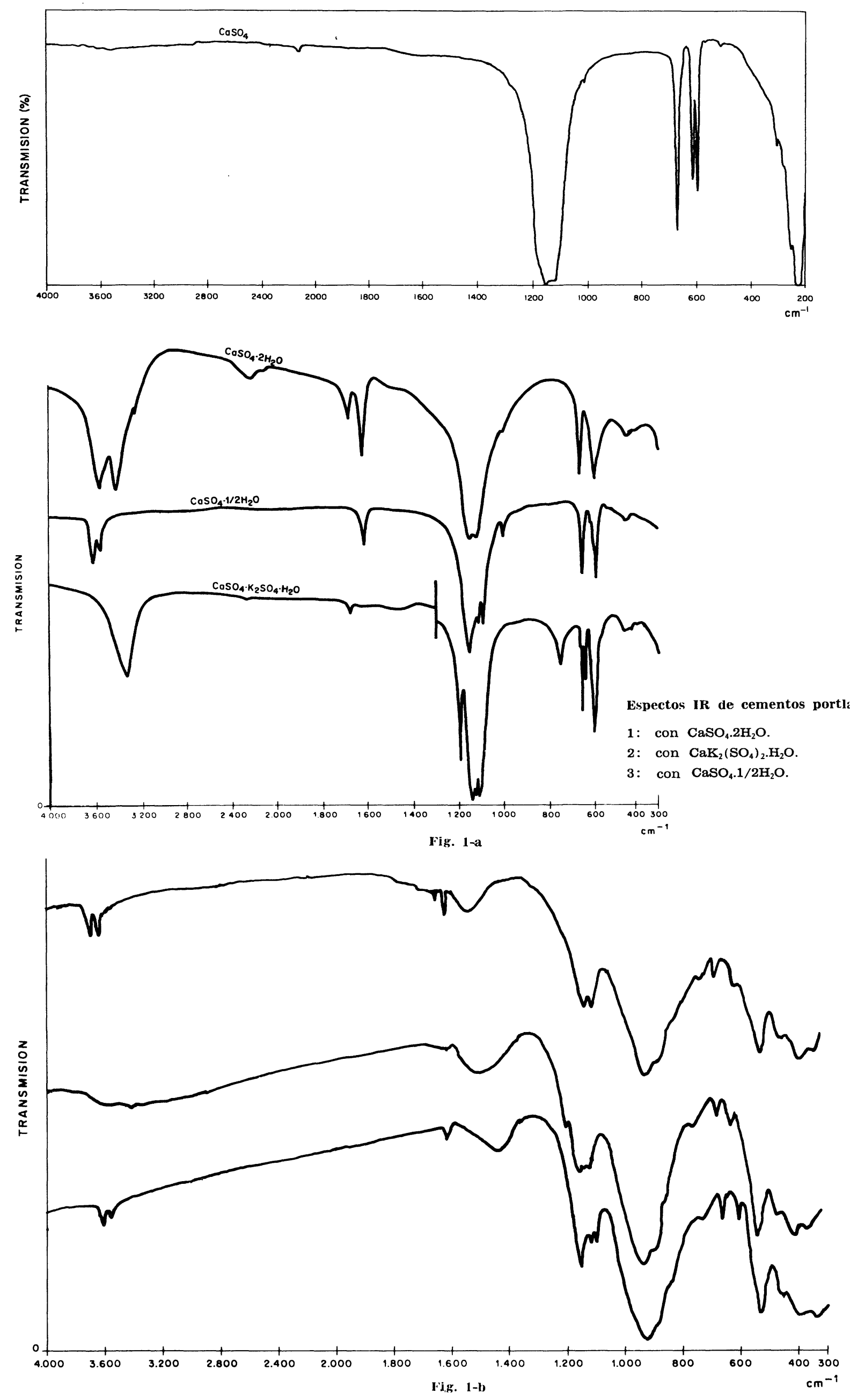


\section{CUADRO 1}

Frecuencias $\left(\right.$ en $\mathrm{cm}^{-1}$ ) y asignaciones del $\mathrm{SO}_{4}{ }^{2-}$ en el $\mathrm{CaSO}_{4} \cdot 2 \mathrm{H}_{2} \mathrm{O} ; \mathrm{CaSO}_{4} \cdot 1 / 2 \mathrm{H}_{2} \mathrm{O} ; \mathrm{CaSO}_{4}$ y $\mathrm{CaK}_{2}\left(\mathrm{SO}_{4}\right)_{2} \cdot \mathrm{H}_{2} \mathrm{O}(15)$

\begin{tabular}{|c|c|c|c|c|c|c|}
\hline & Frecuencia & Asignación & Frecuencia & Asignación & Frecuencla & Asignación \\
\hline $\mathrm{CaSO}_{4} \cdot \mathrm{H}_{2} \mathrm{O}$ & $\begin{array}{l}1.152 \mathrm{~h} \\
1.142 \mathrm{~F} \\
1.115 \mathrm{~F}\end{array}$ & $\nu_{3}$ & $\left.\begin{array}{rl}1.002 \mathrm{D} \\
668 \mathrm{~m} \\
662 \mathrm{~m}\end{array}\right\}$ & $\begin{array}{l}\nu_{1} \\
\nu_{4}\end{array}$ & $\left.\begin{array}{l}468 \mathrm{~d} \\
425 \mathrm{~d} \\
312 \mathrm{~m}\end{array}\right\}$ & $\begin{array}{l}\nu_{2} \\
i ?\end{array}$ \\
\hline $\mathrm{CaSO}_{4} 1 / 2 \mathrm{H}_{2} \mathrm{O}$ & $\begin{array}{l}1.155 \mathrm{~F} \\
1.132 \mathrm{~h} \\
1.118 \mathrm{f} \\
1.094 \mathrm{f}\end{array}$ & $\nu_{3}$ & 1.008 & $\nu_{1}$ & $\left.\begin{array}{l}662 \mathrm{~m} \\
628 \mathrm{D} \\
600 \mathrm{~m} \\
430 \mathrm{D}\end{array}\right\}$ & $\begin{array}{l}y_{4} \\
y_{2}\end{array}$ \\
\hline $\mathrm{CaSO}_{4}$ & $\begin{array}{l}1.158 \mathrm{f} \\
1.130 \mathrm{~F}\end{array}$ & $\nu_{3}$ & & & $\begin{array}{l}675 \mathrm{f} \\
612 \mathrm{f} \\
593 \mathrm{f}\end{array}$ & $\nu_{4}$ \\
\hline $\mathrm{CaK}_{2}\left(\mathrm{SO}_{4}\right)_{2} \cdot \mathrm{H}_{2} \mathrm{O}$ & $\begin{array}{l}1.194 \mathrm{f} \\
1.150 \mathrm{~h} \\
1.140 \mathrm{~F} \\
1.128 \\
1.108 \mathrm{~d}\end{array}$ & $\nu_{3}$ & $\left.\begin{array}{l}752 \mathrm{~d} \\
658 \mathrm{~m} \\
620 \mathrm{~h} \\
604 \mathrm{f} \\
580 \mathrm{~h}\end{array}\right\}$ & $\nu_{1}$ & $\begin{array}{l}470 \mathrm{D} \\
440 \mathrm{D}\end{array}$ & $\nu_{2}$ \\
\hline
\end{tabular}

Naturalmente, la proporción en la cual entran estos compuestos a formar parte del cemento está directamente relacionada con la intensidad relativa de las bandas de absorción específicas. La determinación cuantitativa del yeso se podría valorar por la medida de la absorbancia de la banda en $667 \mathrm{~cm}^{-1}$, la del hemihidrato por la medida de la banda en $660 \mathrm{~cm}^{-1}$ y là de singenita por la banda en $658 \mathrm{~cm}^{-1}$

NIEL (20) propone un método para el estudio cuantitativo de las mezclas (binarias y ternarias) del yeso, hemihidrato y anhidrita. Este método es como sigue: Se miden las absorbancias de las bandas en $667,660,673 \mathrm{~cm}^{-1}$ que corresponden, respectivamente, a $\mathrm{CaSO}_{4}$ dihidrato, hemihidrato y anhidrita. Pero al estar tan próximas estas bandas se solapan mutuamente. Por ello se hacen las medidas de las extinciones relativas $\left(E / E_{\max }\right)$ referidas al máximo de extinción en cada caso. En el cuadro 2 se dan estos valores propuestos por NIEL.

$$
\text { C UADRO } 2
$$

Extinciones relativas de yeso, hemihidrato y anhidrita en la zona de $15 \mu\left(666 \mathrm{~cm}^{-1}\right)$, referidas al máximo de extinción en cada caso

Longitudes de orden

$$
\begin{array}{lll}
14,85 \mu & 15,0 \mu & 15,15 \mu \\
673 \mathrm{~cm}^{-1} & 667 \mathrm{~cm}^{-1} & 660 \mathrm{~cm}^{-1}
\end{array}
$$

\begin{tabular}{|c|c|c|c|}
\hline Sustanclas & & relativ & \\
\hline Anhidrita $\left(\mathrm{CaSO}_{4}\right)$ & 1 & 0,3 & 0 \\
\hline Yeso $\left(\mathrm{CaSO}_{4} .2 \mathrm{H}_{2} \mathrm{O}\right)$ & 0,3 & 1 & 0,35 \\
\hline Hemihidrato $\left(\mathrm{CaSO}_{4} \cdot 1 / 2 \mathrm{H}_{2} \mathrm{O}\right)$ & 0.1 & 0,35 & 1 \\
\hline
\end{tabular}


Con ayuda de esta tabla NIEL establece los sistemas de ecuaciones siguientes:

a) Yeso $y$ hemihidrato

$$
\begin{array}{ll}
\mathrm{g}=\mathrm{G}+0,35 \mathrm{H} & \mathrm{H}=1,14(\mathrm{~h}-0,35 \mathrm{~g}) \\
\mathrm{h}=\mathrm{H}+0,35 \mathrm{C} & \mathrm{G}=1,14(\mathrm{~g}-0,35 \mathrm{~h})
\end{array}
$$

b) Yeso y anhidrita

$$
\begin{array}{ll}
\mathrm{g}=\mathrm{G}+0,3 \mathrm{~A} & \mathrm{~A}=1,1(\mathrm{a}-0,3 \mathrm{~g}) \\
\mathrm{a}=0,3 \mathrm{G}+\mathrm{A} & \mathrm{G}=1,1(\mathrm{~g}-0,3 \mathrm{a})
\end{array}
$$

c) Hemihidrato y anhidrita

$$
\begin{array}{rr}
\mathrm{h}=\mathrm{H}+0,1 \mathrm{~A} & \mathrm{~A}=1,01(\mathrm{a}-0,1 \mathrm{~h}) \\
\mathrm{a}=0,3+0,1 \mathrm{H} & \mathrm{H}=1,01(\mathrm{~h}-0,1 \mathrm{a}) \\
& (\mathrm{h}-0,1 \mathrm{a})
\end{array}
$$

d) Yeso, hemihidrato y anhidrita

$$
\begin{array}{ll}
\mathrm{a}=\mathrm{A}+0,3 \mathrm{G}+0,1 \mathrm{H} & \mathrm{A}=1,12 \mathrm{a}-0,35 \mathrm{~g} \\
\mathrm{~g}=0,3 \mathrm{~A}+\mathrm{G}+0,35 \mathrm{H} & \mathrm{G}=1,25 \mathrm{~g}-0,47 \mathrm{~h}-0,37 \mathrm{a} \\
\mathrm{h}=0,35 \mathrm{G}+\mathrm{H} & \mathrm{H}=1,14 \mathrm{~h}-0,43 \mathrm{a}-0,13 \mathrm{a}
\end{array}
$$

siendo:

$$
\begin{aligned}
& \mathrm{g}=\text { extinción medida en } 15,00 \mu\left(667 \mathrm{~cm}^{-1}\right) \\
& \mathrm{a}=\text { extinción medida en } 14,85 \mu\left(673 \mathrm{~cm}^{-1}\right) \\
& \mathrm{h}=\text { extinción medida en } 15,15 \mu\left(660 \mathrm{~cm}^{-1}\right) \\
& \mathrm{G}=\text { extinción causada por el yeso. } \\
& \mathrm{A}=\text { extinción causada por la anhidrita. } \\
& \mathrm{H}=\text { extinción causada por el hemihidrato. }
\end{aligned}
$$

Una segunda propuesta de NIEL, para determinar el porcentaje de hemihidrato y de yeso mezclados en el cemento anhidro, se basa en la deshidratación parcial del yeso, calentando las muestras durante 1 hora a $120^{\circ} \mathrm{C}$. Con ello se consigue que todo sea hemihidrato, medible por la banda correspondiente de la zona de $9,12 \mu\left(1.100 \mathrm{~cm}^{-1}\right)$. Comparando las medidas de la absorbancia de esta banda, antes y después del calentamiento, se deducirá la cantidad de $\mathrm{CaSO}_{4} .2 \mathrm{H}_{2} \mathrm{O}$ existente originalmente.

También se puede hacer calentando el cemento hasta $450^{\prime \prime} \mathrm{C}$, pasando entonces los sulfatos a anhidrita, y operando como se indicó anteriormente.

En todo caso, con algo de experiencia, se puede estimar de forma inmediata la cantidad aproximada de sulfatos cálcicos que lleva el cemento, con la simple observación del correspondiente espectro IR.

La detección de la singenita en el cernento es de interés, ya que ese compuesto puede dar lugar a "aterronamiento" del cemento anhidro y a alteraciones en el fraguado.

\subsection{Adiciones activas}

El RC-75 (21) establece, entre otros tipos. cementos portland con adiciones activas, cementos siderúrgicos y cementos puzolánicos. 
Los cementos portland con adiciones activas se obtienen por molturación conjunta de clínker de cemento portland y regulador de fraguado, en proporción superior o igual al 80 por ciento en peso y escoria siderúrgica, puzolana o ambas en proporción igual o inferior al 20 por ciento en peso.

Los cementos siderúrgicos llevan como adición activa escoria siderúrgica y los puzolánicos, puzolana. Este tipo de cementos puede ser de dos clases:

- Puzolánico I, que contiene puzolana natural o de cualquier otro tipo excluidas las cenizas volantes, y

- Puzolánico II, que contiene cenizas volantes.

La medida de la puzolanicidad, e incluso su propia naturaleza, son problemas que suscitan polémicas y que aún están sin resolver satisfactoriamente. En opinión del que esto escribe la naturaleza del fenómeno puzolánico podría esquematizarse como sigue:

Una puzolana adsorbe cal a causa de su elevada superficie específica. Paulatinamente esta cal adsorbida entra en reacción principalmente con los silicatos y aluminatos de la puzolana, formando los correspondientes compuestos cálcicos.

Una vez transcurrida esta fase, la puzolana "expulsa" el exceso de cal, es decir, aquella cal no combinable al haberse formado ya los compuestos estequiométricos correspondientes.

Es comprensible entonces que, según esta hipótesis, la puzolanicidad tenga dos facetas consecutivas: En la primera, la retención de la cal depende de la superficie específica de la puzolana. En un segundo paso, la cal es definitivamente "retenida" al combinarse con los aluminatos y silicatos principalmente de la puzolana. Por ello una puzolana será tanto más activa cuanto esos compuestos sean menos cristalinos, más amorfos y con más alteraciones y defectos reticulares. El papel de los álcalis es importante por su facilidad de inclusión en redes cristalinas, y su papel de "deformadores".

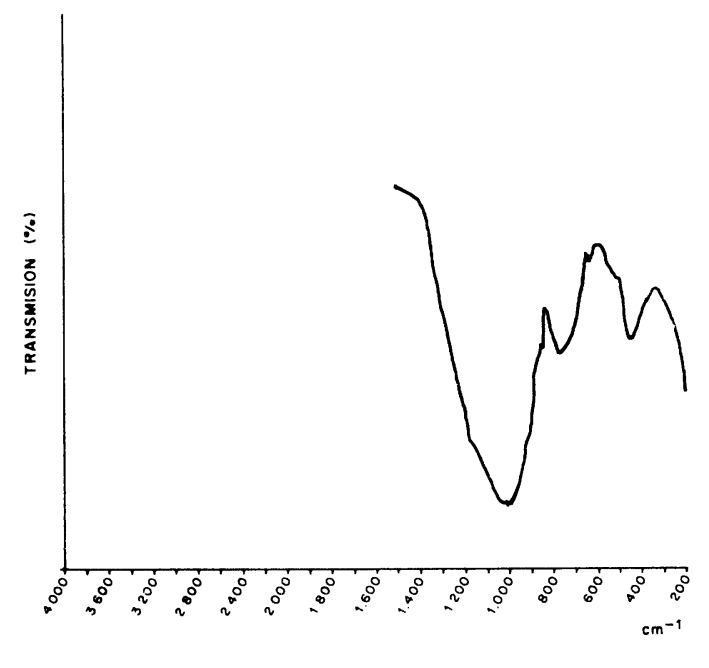

Fig. :

4.2.1. Espectro IR de una ceniza (fig. 2)

En el espectro de la ceniza se observan diversas bandas características de una sustancia 
con estructuras muy desordenadas. Por su forma y por la frecuencia de absorción a que aparecen se deduce que son debidas a sílice vítrea. La banda continua y difusa que aparece entre 650 y $800 \mathrm{~cm}^{-1}$ es causada, principalmente, por tetraedros $\mathrm{AlO}_{4}$ deformados. En las proximidades de $800 \mathrm{~cm}^{-1}$ también hay bandas de tetraedros $\mathrm{SiO}_{4}$.

No se observan bandas específicas de octaedros $\mathrm{AlO}_{6}$ (que, si están aislados, darían bandas por debajo de $650 \mathrm{~cm}^{-1}$ ). Esto se interpreta en el sentido de considerar que todo el $\mathrm{Al}$ estará tetracoordinado. A su vez esto supone que es capaz de reforzar las estructuras vítreas en las que se introduce en cantidad moderada, o incluso actuar como formador de red (5).

Como una primera aproximación puede esperarse de la ceniza en estudio un buen comportamiento puzolánico, derivado, principalmente, del desorden estructural y de la coordinación tetraédrica del $\mathrm{Al}$.

El análisis químico de la ceniza es el siguiente:

$$
\begin{array}{llll}
\text { P.F. }=8,62 & \mathrm{SiO}_{2}=47,30 & \mathrm{Fe}_{2} \mathrm{O}_{3}=6,74 & \mathrm{MgO}=2,39 \\
\text { R.I. }=0,18 & \mathrm{Al}_{2} \mathrm{O}_{3}=28,28 & \mathrm{CaO}=3,27 & \mathrm{SO}_{3}=0,42
\end{array}
$$

\subsubsection{Espectro IR de una escoria (fig. 3)}

La muestra empleada para realizar el presente estudio se trata de una escoria de alto horno, cuyo análisis químico es el siguiente:

$$
\begin{array}{lll}
\text { P.F. }=0,51 & \mathrm{Fe}_{2} \mathrm{O}_{3}=7,39 & \mathrm{SO}_{3}=0,09 \\
\text { R.I. }=0,42 & \mathrm{CaO}=28,84 & \mathrm{MnO}=2,50 \\
\mathrm{SiO}_{2}=40,18 & \mathrm{MgO}=13,04 & \\
\mathrm{Al}_{2} \mathrm{O}_{3}=4,77 &
\end{array}
$$

Este tipo de escoria se está empleando industrialmente como adición activa en el cemento.

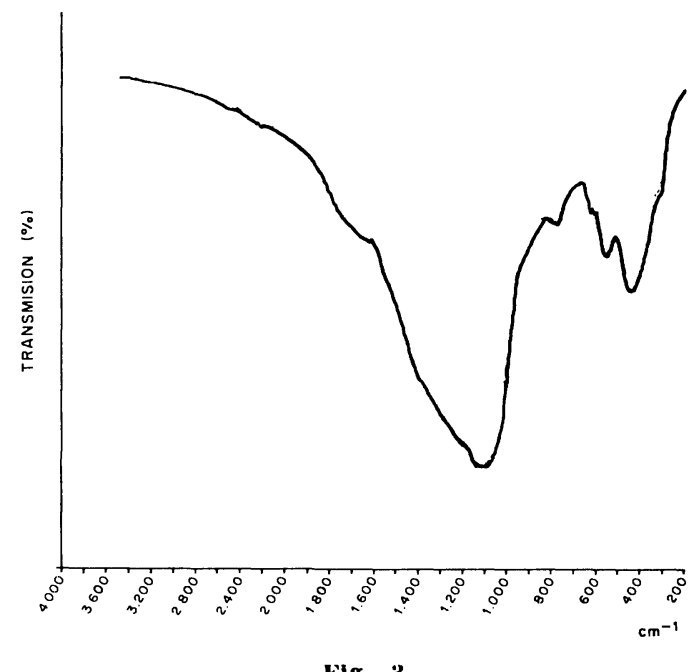

Fig. 3

En su espectro IR (fig. 3) destacan las bandas características de tetraedros $\mathrm{SiO}_{4}(1.050-1.150$ alrededor de $800 \mathrm{~cm}^{-1}$ y en $\left.470-450 \mathrm{~cm}^{-1}\right)$. Hay bandas de $\mathrm{AlO}_{4}\left(700-800 \mathrm{~cm}^{-1}\right)$ y de $\mathrm{FeO}_{4}(600-$ $700 \mathrm{~cm}^{-1}$ ). Además aparece una banda en $555 \mathrm{~cm}^{-1}$ que atribuimos a uniones $\mathrm{SiO}_{4}-\mathrm{AlO}_{6}$, lo que supone un cierto grado de cristalinidad.

El espectro indica un cierto orden estructural muy superior al existente en la ceniza. 


\subsubsection{Espectro IR de una puzolana (fig. 4)}

Un estudio bastante amplio de algunas puzolanas naturales españolas ya ha sido realizado en este Instituto Eduardo Torroja (22). No obstante, y siguiendo la línea del presente trabajo, se da a continuación el espectro IR de una 'uzolana natural utilizada industrialmente, así como nuestra interpretación del mencionado espectro.

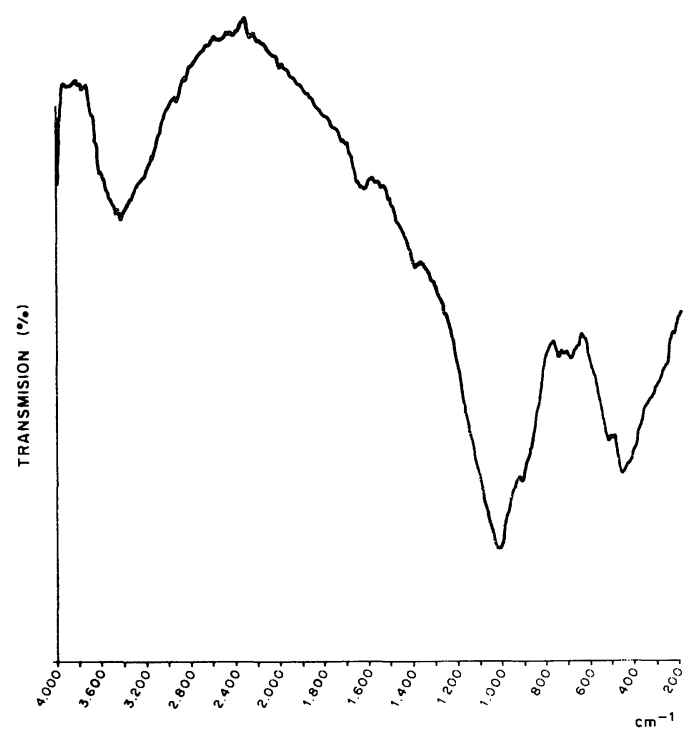

Fig. 4

El análisis químico de la puzolana estudiada es el siguiente:

$$
\begin{array}{ll}
\text { P.F. }=0,42 & \mathrm{CaO}=10,00 \\
\mathrm{SiO}_{2}=44,20 & \mathrm{MgO}=0,20 \\
\text { R.I. }=0,17 & \mathrm{SO}_{3}=0,02 \\
\mathrm{Al}_{2} \mathrm{O}_{3}=26,00 & \mathrm{Na}_{2} \mathrm{O}=2,08 \\
\mathrm{Fe}_{2} \mathrm{O}_{3}=4,80 & \mathrm{~K}_{2} \mathrm{O}=1,08 \\
& \mathrm{MnO}=0,13
\end{array}
$$

En el espectro se observan bandas en las zonas de 3.400 y $1.600 \mathrm{~cm}^{-1}$ respectivamente. Son causadas por vibraciones de valencia y deformación de agua adsorbida.

En la región de $1.000 \mathrm{~cm}^{-1}$ aparecen las bandas de mayor intensidad del espectro, que corresponden a vibraciones de valencia $\nu_{3} \mathrm{SiO}_{4}$. Son debidas a la sílice amorfa.

La absorción difusa y continua, entre 850 y $950 \mathrm{~cm}^{-1}$, la interpretamos como causada por tetraedros $\mathrm{SiO}_{4}$ y $\mathrm{AlO}_{4}$.

La absorción que se ve entre 700 y $800 \mathrm{~cm}^{-1}$ es debida principalmente a $\mathrm{AlO}_{4}$. También los tetraedros $\mathrm{FeO}_{4}$ influyen en esta zona del espectro.

La región de alrededor de $540 \mathrm{~cm}^{-1}$ indica uniones $\mathrm{SiO}_{4}-\mathrm{AlO}_{6}$. Aun cuando este tipo de uniones indica un cierto grado de cristalinidad de la muestra, la banda ancha y poco definida se interpreta como causada por uniones con desorden estructural.

A más baja frecuencia (hacia $450 \mathrm{~cm}^{-1}$ ) aparecen bandas de $\nu_{4}-\mathrm{SiO}_{4}$. 
En la figura 5 se dan los espectros de cementos que contiene una relación puzolana/clínker + yeso de $40 / 60 ; 60 / 40 ; 70 / 30$ y $80 / 20$, respectivamente.

Las diferencias que se observan en los espectros resultantes vienen dadas, lógicamente, por las zonas de absorción tanto de la puzolana como del clínker y yeso.
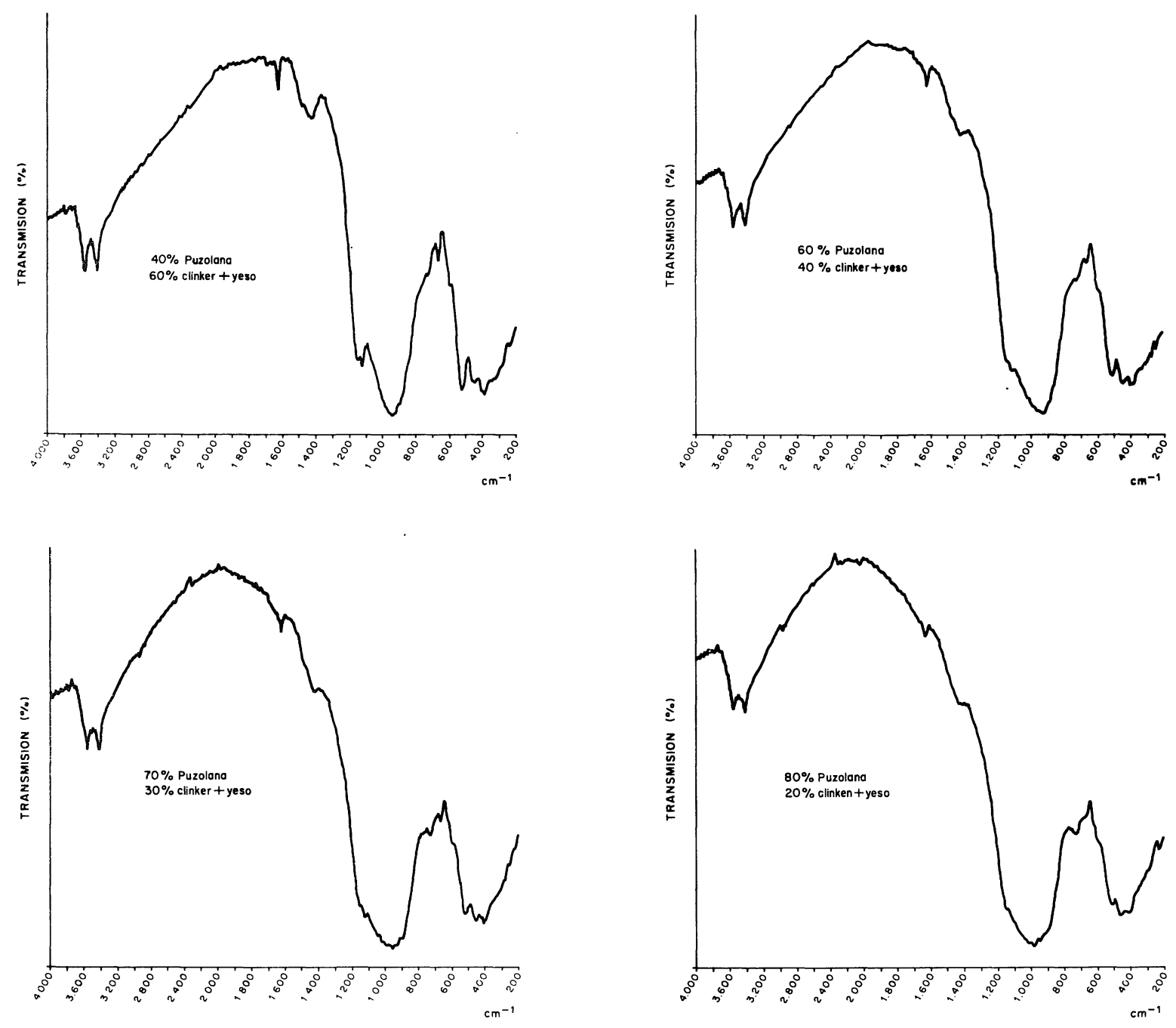

Fig. :

En esas zonas del espectro se destaca el que, a medida que el cemento contiene una mayor proporción de puzolana, va aumentando la anchura de la banda que aparece en la región de $920 \mathrm{~cm}^{-1}$, hasta tal punto que llega a enmascarar las correspondientes a $\mathrm{SO}_{4}$ en 1.110 $1.150 \mathrm{~cm}^{-1}$ ). La amplitud de esta "banda" (que es realmente la superposición de varias) se "extiende" hacia más altas frecuencias, con mayor intensidad cuanto más puzolana contiene la muestra.

La zona de 700-800 $\mathrm{cm}^{-1}$ aparece claramente influida por la cantidad de puzolana de la muestra; la absorción causada por uniones Al-O se hace cada vez más difusa, de tal forma que si se unieran los mínimos de absorción en 690 y $790 \mathrm{~cm}^{-1}$ con una línea recta, la pendiente correspondiente iría en disminución a medida que la muestra estudiada contuviera mayor cantidad de puzolana. 
Este efecto podría aprovecharse para determinar en forma relativa la cantidad de clínker y puzolana que contiene un cemento, en el estudio de muestras que poseen clínker y puzolana de la misma naturaleza.

Asimismo, se observa en la zona de bajas frecuencias que las bandas se van haciendo menos netas a medida que la cantidad de puzolana contenida en el cemento aumenta.

\subsubsection{Estudio de cementos con diversas proporciones de escoria y clínker}

En la figura 6 se dan los espectros de cementos que contienen una relación escoria/clínker + yeso de $40 / 60 ; 60 / 40 ; 70 / 30$ y $80 / 20$, respectivamente.
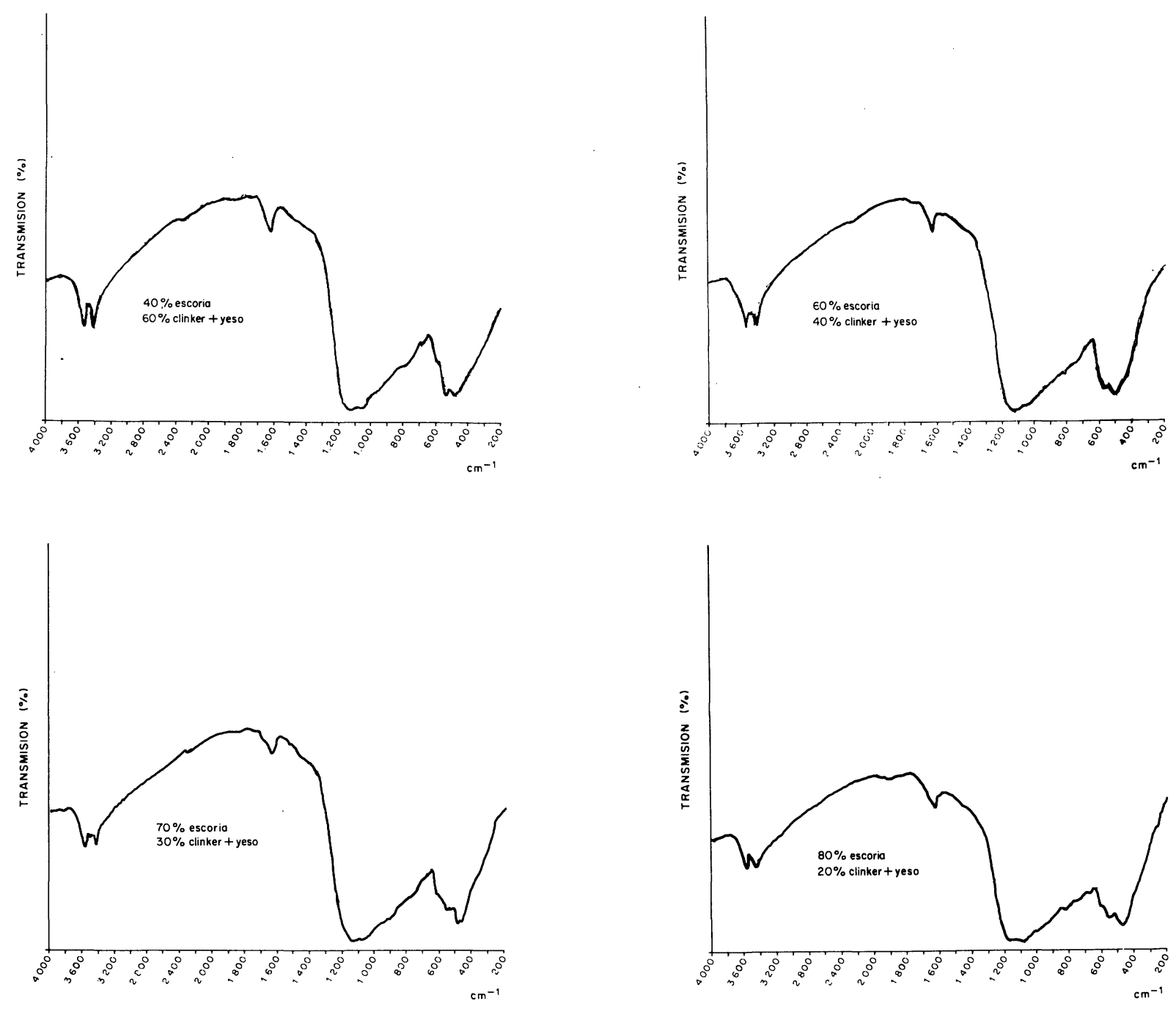

Fig. 6

No se observan con nitidez diferencias entre los espectros IR de los cementos estudiados. Las distintas proporciones de escoria en relación a clínker + yeso quizá se puedan estimar a partir de la "disminución" aparente de la intensidad de la banda en la zona de 520-540 $\mathrm{cm}^{-1}$, a medida que la proporción de la escoria aumenta en el cemento. Esta "disminución" es causada tanto por el solapamiento que sufre a consecuencia de la absorción producida por la escoria en aquella región del espectro, como, lógicamente, por la disminución de la cantidad de clínker de las muestras correspondientes. 
De la misma forma que en el caso de los cementos con puzolana, se observa aquí que a medida que la muestra contiene mayor cantidad de adición activa, las hipotéticas líneas que unieran los mínimos de absorción en 690 y $790 \mathrm{~cm}^{-1}$ de los espectros correspondientes, disminuirán su pendiente.

\subsection{Conclusiones prácticas}

- Por espectroscopía IR se identifica con facilidad el tipo de sulfato que posee el cemento como regulador de fraguado.

- Se puede determinar cuantitativamente, mediante fórmulas propuestas por NIEL, las distintas proporciones de yeso, hemihidrato y anhidrita que puede contener un cemento.

- Por espectroscopía IR se pueden estudiar distintas adiciones activas (puzolana natural, cenizas y escorias) sea cual fuere su grado de cristalinidad.

- Por espectroscopía IR es posible clasificar cementos fabricados con un mismo clínker y una misma puzolana (o incluso una misma escoria), mezclados en proporciones diferentes, según la proporción de adición activa que contenga.

\section{B I B L I O G R A F I A}

(1) FARmer, V. C.: European Spectroscopy News. p. 25 n.” 25 (1979).

(2) Morcillo, J. y MAdroñero, R.: “Aplicación práctica de la espectroscopía infrarroja”. Facultad de Ciencias. Universidad de Madrid (1962).

(3) Nakamoto, K.: "Infrared spectra of Inorganic and Coordination Compounds". Joh Wiley and Sons. Inc. New York-London, pág. 91 (1965).

(4) Font-Altaba, M.: “Atlas de Mineralogía”. Ed. Jover (1974).

(5) Chaussidon: Bull. Soc. Franç. Ceram. A 532, pág 57 (1967).

(6) TARTE, P.: "Étude experimentale et interpretation du spectre infra-rouge des silicates et des gemanates. Application à des problemes structuraux relatifs á l'etat solide”. Acad. Royale de Belgique. Tomo XXXV, fasc. 4. a pág. 35. (1965).

(7) Hidalgo, A.: Espectroscopía IR de minerales de la arcilla. Instituto de Optica "Daza de Valdés" C.S.I. Madrid.

(8) FArmer, V.: Clay Min 7, pág. 373 (1968).

(9) Eitel, W.: Silicate Science. Academic Press. London New York (1964).

(10) TARTE, P.: Spectrochimica Acta. Vol. 23 A. P. 2127 (1967).

(11) Rocchiccioli, C.: Chimie Analitique, n.' 46, p. 452 (1964).

(12) Volant, J.: Tesis Doctoral. París (1964).

(13) Guinier, A. y Regourd, M.: Ciments et betons, núms. 643-644-645-646 (1969).

(14) Yamaguchi, G. y Uchikawa, H.: Semento Gijutsu Nenpa XVI, p. 23 (1962).

(15) Bensted, J. y Varma, S. P.: Cement Technology. pág. 378 julio-agosto (1979).

(16) VAzQUez, T.: Tesis doctoral, Facultad de Ciencias Químicas. Universidad Complutense. Madrid (1975).

(17) TARte, P.: Revue de Chimie Minerale t 1 p. 425 (1964).

(18) TARTE, P.: Silicates Industriels, n. ${ }^{\circ} 31$, pág. 343 (1966).

(19) Vazquez, T.: Materiales de Construcción, n.` 161 (1976).

(20) Kassner, B. y Henning, O.: Silikattechnik, pág. 23, n.” 4 (1972) y pág. 24, n. 1 (1972).

(21) Niel, E.: Tesis Doctoral. Auchen (1962).

(22) R.C.-75: Pliego de Prescripciones Técnicas Generales para la Recepción de Cemento M.O.P. (1975).

(23) Luxan, M. P.: Tesis Doctoral. Facultad de Ciencias Químicas. Universidad Complutense. Madrid (1975). 